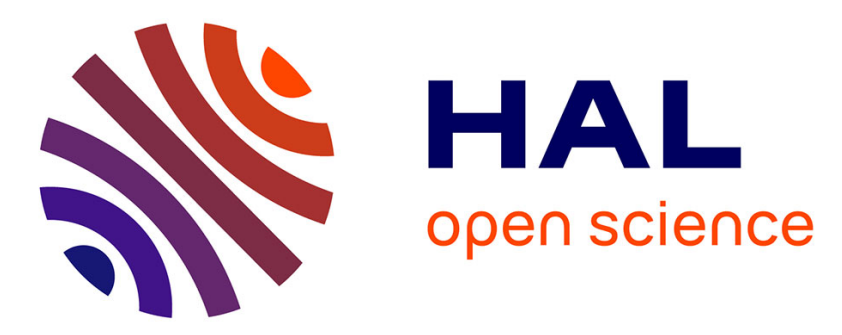

\title{
Lorsque gérer l'action sociale devient affaire d'action collective. Une contribution à l'analyse des partenariats dans l'action sociale territorialisée
}

Gilles Frigoli

\section{- To cite this version: \\ Gilles Frigoli. Lorsque gérer l'action sociale devient affaire d'action collective. Une contribution à l'analyse des partenariats dans l'action sociale territorialisée. Revue française des affaires sociales, 2004, 4, pp.85-103. halshs-00079012}

\section{HAL Id: halshs-00079012 \\ https://shs.hal.science/halshs-00079012}

Submitted on 25 Oct 2006

HAL is a multi-disciplinary open access archive for the deposit and dissemination of scientific research documents, whether they are published or not. The documents may come from teaching and research institutions in France or abroad, or from public or private research centers.
L'archive ouverte pluridisciplinaire HAL, est destinée au dépôt et à la diffusion de documents scientifiques de niveau recherche, publiés ou non, émanant des établissements d'enseignement et de recherche français ou étrangers, des laboratoires publics ou privés. 


\title{
Lorsque gérer l'action sociale devient affaire d'action collective Une contribution à l'analyse des partenariats dans l'action sociale territorialisée
}

\author{
Le cas de la lutte contre l'exclusion
}

\author{
Gilles Frigoli*
}

L'action publique locale est le lieu d'un développement spectaculaire du partenariat comme principe d'action. Il n'est guère aujourd'hui de domaines de l'action publique qui échappent à cette évolution que l'on peut lier à un processus plus global de recomposition des modes d'intervention étatique. L'essor de politiques publiques qui tendent à accroître l'autonomie des acteurs locaux et valorisent leur capacité à concevoir des solutions collectives et négociées à des problèmes appréhendés comme transversaux, en témoigne ${ }^{1}$.

Plusieurs facteurs concourent à conférer à la lutte contre l'exclusion (et plus généralement au «social » au sens large) un statut particulier qui explique qu'elle soit souvent convoquée pour illustrer l'ampleur du phénomène et en repérer les enjeux, notamment en raison du nombre considérable de dispositifs de coordination qui se sont succédé ces dernières années dans le champ de l'action sociale territorialisée. Dans la période récente, on aurait peine à trouver en ce domaine un programme public qui n'ait été conçu et organisé sur la base d'un appel à la coopération des divers acteurs concernés par les problèmes à régler ${ }^{2}$. De plus, il faut noter le rôle joué par les « politiques de la ville » dans l'orientation de l'action de l'État vers une gestion partenariale des territoires à partir d'un découpage fondé sur l'élaboration de projets, l'identification de problèmes ou de populations spécifiques. Pour plusieurs

\footnotetext{
* Maître de conférences en sociologie à l'université de Nice-Sophia-Antipolis et chercheur au sein du SOLIIS-URMIS (UMR CNRS 7032).

1 Voir à ce sujet les analyses convergentes sur ce point de Gaudin (1996), Duran et Thœnig (1996), Lascoumes (1996), concernant ce type de politiques publiques que ces auteurs qualifient respectivement de « contractuelles », « constitutives », « d'organisation ».

2 On pourrait citer, parmi une liste extrêmement longue, le comité départemental de la politique de la ville, le conseil départemental pour le logement des personnes défavorisées, le comité de coordination des politiques de lutte contre les exclusions, le comité départemental $d u$ Fonds solidarité logement, le conseil départemental d'insertion, etc. Notons que le «plan de cohésion sociale », présenté en Conseil des ministres le 30 juin 2004 prévoit la création d'une nouvelle instance au sein de ce paysage déjà complexe : la "charte de cohésion sociale », « instrument proposé aux acteurs locaux qui souhaiteront s'engager dans la réalisation d'un projet territorial déclinant et incarnant les différents axes du plan de cohésion sociale ». Voir le dossier de presse du site internet du ministère de l'Emploi, du Travail et de la Cohésion sociale : www.cohesionsociale.gouv.fr.
} 
raisons, ces politiques dites « transversales » constituent dans leurs versions successives une série de cas d'école qui n'ont pas manqué d'être largement commentés par la presse et analysés par les chercheurs. On pourrait, à ce titre, évoquer la médiatisation massive du « problème des quartiers » et des plans d'action qui se sont succédé (Champagne, 1993) ; l'instrumentalisation par la plupart des partis politiques, à laquelle a donné lieu l'émergence du thème de la banlieue (Wacquant, 1992) ; la diversité des acteurs impliqués et la complexité des montages institutionnels correspondants, de l'interministérialité à l'intercommunalité (Dammame, Jobert, 1995); le caractère transversal de l'appréhension des problèmes à traiter dans le cadre du « développement social » (Guyot, Laudoyer, 1998) ; l'implication, enfin, des élus locaux dans les divers dispositifs (Ion, 1990).

Par ailleurs, l'action sociale est sans doute le domaine dont l'architecture a été la plus profondément bouleversée par des lois de décentralisation qui ont constitué un moment charnière dans cette évolution en direction de modes d'intervention étatique valorisant la mise en place de partenariats locaux. L'action sociale constitue en effet un des domaines les plus directement touchés par les «transferts de compétence » issus des lois de décentralisation. Parmi les nombreuses compétences que se sont vu attribuer les conseils généraux dans le cadre de la première décentralisation (protection de l'enfance, protection maternelle et infantile, gestion des foyers de vie et des maisons de retraite, etc.) on retiendra surtout la gestion du service social départemental, aujourd'hui au cœur de la plupart des partenariats qui se mettent en place dans la lutte contre l'exclusion ${ }^{1}$. De même, la loi 20031200 du 18 décembre 2003 portant décentralisation en matière de revenu minimum d'insertion (RMI) et création du revenu minimum d'activité, a confié au département le pilotage du RMI et le projet de loi relatif aux libertés et responsabilités locales ${ }^{2}$ prolonge dans son titre III, ce mouvement de transfert de compétences, notamment en matière de « coordination des dispositifs et services qui concourent à l'insertion et à l'action sociale » (article 29).

En outre, il existe un lien direct entre le développement de l'approche partenariale dans l'ensemble des domaines de l'action publique et l'inscription des thèmes de la solidarité et de l'exclusion sur les agendas gouvernementaux au cours des vingt dernières années (Behar, 2001).

Enfin, l'aide en faveur des personnes en difficulté constitue un des domaines dans lesquels les débats quant au rôle qui devrait être celui de l'État sont, depuis fort longtemps, les plus virulents. Il n'est donc guère étonnant que l'émergence du partenariat comme forme de construction de l'action

$\overline{1}$ Voir parmi une bibliographie pléthorique, les deux bilans dressés par l'ODAS respectivement dix ans et vingt ans après la première décentralisation : C. Padieu, J.-L. Sanchez (1994), ODAS (2003).

$2 \mathrm{Au}$ moment où nous écrivons ce papier, le projet a été adopté en deuxième lecture par le Sénat le $1^{\text {er }}$ juillet 2004. 
sociale suscite des prises de position normatives, soit que l'on y associe l'hypothèse d'un retrait de l'État dans la gestion de la pauvreté, soit que l'on dénonce le fait qu'elle s'accompagnerait du développement de politiques sociales d'inspiration «néolibérale » ciblées sur les catégories les plus défavorisées de la population (Borgetto, 2003).

Ces différents éléments concourent à conférer au domaine de l'action sociale un statut particulier justifiant que l'on en fasse une illustration particulièrement éclairante du succès de ce mode d'action et des enjeux qu'il soulève.

Toutefois, à notre sens, deux éléments supplémentaires qui ont, jusqu'ici, fait l'objet d'une faible attention dans la littérature consacrée aux partenariats locaux dans l'action sociale, doivent être pris en compte. Le premier concerne le caractère " indivisible » des problèmes qu'au nom de la lutte contre les exclusions, les acteurs locaux entreprennent de gérer, ainsi que le poids de cette « indivisibilité » dans le renforcement de l'interdépendance de ces acteurs. Le second a trait à la diversité des lignes d'argumentation des textes officiels, émanant des instances étatiques, qui justifient le recours à la démarche partenariale. Leur prise en compte nous semble importante car, nous en faisons l'hypothèse, leur conjonction - parce qu'elle a pour effet d'injecter de l'incertitude dans les « systèmes d'action territoriaux » qui ont à organiser leur coopération interne - contribue à favoriser ce que Friedberg (1993) nomme l'«autonomisation des échanges politiques » entre partenaires. Il faut entendre par-là la mise en place de partenariats « fonctionnant sans principe de réalité autre que des considérations de pouvoir et d'influence des parties prenantes » (op. cit., p. 132-133), la négociation des règles de l'échange entre partenaires prenant le pas sur la recherche de solutions négociées aux problèmes que rencontrent les populations, pour devenir en soi le principe régulateur de la coopération.

Afin d'étayer cette hypothèse, nous nous appuierons principalement sur deux enquêtes réalisées dans un département du sud de la France et consacrées à l'étude de dispositifs ayant une forte dimension partenariale : le Fonds d'urgence sociale (FUS) et la Commission de l'action sociale d'urgence (CASU) ${ }^{1}$. Toutefois, notre propos se fonde moins sur l'analyse de ces dispositifs proprement dits que sur la connaissance des dynamiques de la politique menée localement en matière d'action sociale à laquelle leur étude nous a permis d'accéder, notamment sur le dispositif du RMI et sur les acteurs qui le gèrent et qui ont été fortement interpellés par la mise en

1 Le premier dispositif, ponctuel et consécutif aux mouvements de chômeurs de fin 1997, consistait à distribuer localement des prestations financières exceptionnelles aux «personnes et aux familles en situation de détresse grave [...] » (circulaire du 12 janvier 1998 relative au développement du Fonds et des missions d'urgence sociale). Le second, pérenne, vise à « assurer la coordination des dispositifs susceptibles d'allouer des aides, notamment financières, aux personnes et aux familles rencontrant de graves difficultés » - article 154 de la loi du 29 juillet 1998 relative à la lutte contre les exclusions -. (voir à ce sujet le numéro 1-2001 de la Revue française des Affaires sociales consacré aux résultats des différentes études sur site de ces dispositifs). 
œuvre du FUS et de la CASU. Le dispositif des CASU ayant pour objet de favoriser la coordination des actions menées au niveau départemental en faveur des personnes en difficulté, constitue en effet un bon analyseur des logiques déployées par les acteurs locaux dès lors qu'est ouverte la possibilité, ou que s'impose la nécessité, de réorganiser les liens qui fondent la coopération entre les acteurs qui s'inscrivent dans le champ de la lutte contre l'exclusion.

Pour cette raison, en parlant d' « action sociale locale » au sens large, nous ne faisons pas référence à la classification généralement utilisée par les spécialistes du droit de l'aide et de l'action sociales ${ }^{1}$ et qui distingue les droits ouverts par des cotisations (assurances sociales), les droits fondamentaux liés à l'expression de besoins (aide sociale), ou le devoir de solidarité que s'imposent certaines institutions, organismes ou associations (action sociale). Nous désignons une configuration d'acteurs, au sein de laquelle, par-delà la diversité des appartenances institutionnelles, les protagonistes s'identifient mutuellement comme membres d'un même domaine de l'action publique locale. Nous faisons référence à l'existence d'un « noyau dur » d'acteurs locaux unis par des liens d'interconnaissance et de dépendance mutuelle aux différents échelons hiérarchiques et que l'on retrouve généralement dans la plupart des dispositifs ayant trait à la lutte contre 1'exclusion : directions départementales des affaires sanitaires et sociales (DDASS), services sociaux du conseil général, représentants locaux des organismes de protection sociale, etc. ${ }^{2}$

\section{- Des acteurs interdépendants dans la gestion de problèmes aux frontières floues}

La dépendance mutuelle qui lie les acteurs locaux de l'action sociale est en premier lieu à mettre au compte de la multiplication des dispositifs qui, par voie législative ou réglementaire, leur imposent de coordonner leurs interventions, l'engagement à coopérer se manifeste par l'adoption de procédures contractuelles sanctionnées par la signature de conventions entre les partenaires ${ }^{3}$. Mais l'accroissement des interdépendances trouve également sa source dans la transversalité des problèmes à gérer, ou pour reprendre la formulation d'Aldrich (1976), dans leur indivisibilité, au sens où aucun acteur,

\footnotetext{
1 Voir par exemple Thévenet (1997).

2 Cette approche recoupe celle que propose l'IGAS dans un rapport récent consacré au bilan de la loi du 29 juillet 1998 relative à la lutte contre les exclusions : «La sphère de l'action sociale [...] inclut notamment l'action sociale facultative des collectivités territoriales, l'action sociale des organismes de protection sociale, et la mise en œuvre des programmes nationaux de lutte contre les exclusions, généralement impulsés par l'État en partenariat avec les acteurs très divers de l'action sociale » (IGAS, 2004, p. 18).

3 Le débat n'est pas clos concernant le statut juridique des contrats en question. Voir à ce sujet Marcou (1996).
} 
fût-il l'État, ne peut prétendre les résoudre seul. Les problèmes que prend en charge l'action sociale s'offrant à de multiples définitions, il n'est pas d'acteur qui puisse en revendiquer la propriété. Dès lors, le comportement de chacun se trouve contraint par celui de l'ensemble de ceux qui seront en mesure de justifier de leur statut de « copropriétaire », pour peu qu'ils parviennent à imposer comme pertinent et légitime le mode de formulation des problèmes qu'ils mettent en avant. Le cas du RMI illustre bien ce lien entre le caractère indivisible des problèmes et la dépendance mutuelle dans laquelle se trouvent les acteurs engagés dans la négociation de leur qualification ${ }^{1}$.

\section{Les interdépendances formelles dans la gestion du RMI}

Sans entrer dans la description de l'architecture départementale du RMI telle que la prévoyait la loi du $1^{\text {er }}$ décembre $1988^{2}$, on rappellera que le dispositif multiplie les partenaires officiels entre lesquels il instaure des liens de dépendance mutuelle forts. Ainsi en est-il pour tout ce qui concerne la gestion de la prestation monétaire, qui fait appel à des institutions différentes pour assurer la réception et l'instruction des demandes (centres communaux d'action sociale-CCAS -, services départementaux d'action sociale, associations agréées), calculer le montant du RMI (caisses d'allocations familiales - CAF - et Mutualité sociale agricole - MSA -), décider de l'attribution de l'allocation (préfecture). L'offre d'insertion, que la loi impose au conseil général de financer et de garantir aux usagers dès lors qu'ils sont éligibles à la prestation, est quant à elle assurée par des acteurs de statut très divers : associations, sociétés de transport, auto-écoles, crèches, offices municipaux de la jeunesse, CCAS, cabinets de consultants, foyers de jeunes travailleurs, centres hospitaliers, etc. ${ }^{3}$. Enfin, la politique locale d'insertion, que le Conseil départemental d'insertion a pour mission de conduire dans le cadre du dispositif RMI est officiellement coproduite par le préfet et le président du conseil général, la loi n'instaurant pas en la matière de lien hiérarchique entre l'un et l'autre. On retiendra des relations qu'entretiennent les services déconcentrés de l'État et le conseil général qui sont liés par convention dans une quantité de dispositifs, que la coopération

\footnotetext{
1 L'enquête sur laquelle s'appuie cet article, a été menée avant la réforme de 2003 qui confie le pilotage intégral du RMI au conseil général. L'architecture du dispositif d'insertion départemental est en cours d'évolution et l'analyse reste à faire des conséquences de la réforme sur le paysage local en matière d'insertion. Toutefois, si nos données sont de ce fait « datées », que le lecteur nous permette de penser que la démonstration ne l'est pas. Nous nous intéressons moins au RMI en tant que tel qu'à certains processus auxquels la création de ce dispositif a pu donner lieu et aux hypothèses de recherche plus générales que l'on peut en tirer concernant le partenariat local comme modalité de conception et de mise en œuvre des politiques sociales.

2 On pourra pour cela se reporter à la présentation, très claire, que propose l'ouvrage de Valérie Löchen (2000).

3 Cette liste, non exhaustive, est tirée de L'offre d'insertion, guide pratique à l'usage des instructeurs du RMI, manuel conçu et diffusé par les services du conseil général du département concerné par notre enquête. On y recense plus de soixante dispositifs d'insertion (qui correspondent à autant de partenaires impliqués) que peuvent mobiliser les travailleurs sociaux en charge du suivi des allocataires du RMI.
} 
qu'ils sont amenés à établir dans le cadre du RMI puise aux rapports de force qui les lient dans l'organisation de l'action sociale locale et que se confirme ainsi leur statut d' " associés rivaux » qui peuvent d'autant mieux développer leurs stratégies que l'organisation des actions d'insertion à mettre en œuvre et le choix des prestataires de services correspondants relèvent strictement de l'échelon local ${ }^{1}$. On ne s'étendra pas sur les dépendances croisées qui traversent les relations qu'entretiennent les «grands partenaires de l'État » qui composent le noyau dur de l'action publique locale en matière d'action sociale (CAF, caisses primaires d'assurance maladie, Agence nationale pour l'emploi, etc.). Se trouver associé au RMI, c'est disposer des ressources financières que l'État alloue aux participants. C'est aussi être au cœur de l'action sociale locale et satisfaire au principe selon lequel il convient d'être présent dans tous les lieux où l'avenir se négocie, où de nouvelles dispositions pourraient être décidées : c'est « être dans le jeu ».

Plus intéressant est le cas des associations car, pour ces dernières, « être dans le jeu » constitue un enjeu vital au sens propre du terme. Une association d'action sociale qui ne serait pas « missionnée », « agréée », et donc financée pour mener telle ou telle action, serait rapidement condamnée. Etre associé à un dispositif comme le RMI, par exemple pour assurer l'instruction des demandes et le suivi des usagers ou pour leur proposer des « outils d'insertion », c'est bénéficier d'une quasi-rente de situation en matière de financement et d'un socle de légitimité permettant d'étendre son implication dans l'action sociale locale à d'autres activités, donc de se rendre disponible pour d'autres financements mis en circulation sur le « marché public des subventions ». Notons cependant que les associations ne sont pas démunies de ressources dans leur relation avec les financeurs institutionnels tels que l'État, les collectivités territoriales ou les organismes de protection sociale. En premier lieu, certaines d'entre elles ont su se rendre indispensables dans certains domaines d'activité dont les institutions publiques, souvent pour des raisons liées à des contraintes matérielles, se détournent. L'État local, la DDASS en particulier, se trouve souvent aujourd'hui dans l'obligation de faire appel à des associations lorsqu'il entreprend d' " aller sur le terrain », c'est-à-dire d'organiser la rencontre concrète entre la politique publique et l'usager. L'administration sociale ne disposant en effet que de très peu de travailleurs sociaux, les acteurs associatifs constituent souvent les bras de la DDASS et, ce qui est stratégiquement plus lourd d'enjeux, ses yeux. En effet, ce sont dans une assez large

1 Le contenu de la convention est élaboré localement ; par ailleurs, toute une série de conventions complémentaires peut venir la compléter dans le cadre de la politique d'insertion qu'ils ont reçu mission de conduire. Même si des « incitations » à s'engager dans telle ou telle direction leur sont régulièrement adressées par les services centraux du ministère des Affaires sociales par voie de circulaire le plus souvent, on soulignera l'autonomie relative dont disposent les acteurs locaux dans ce domaine. Les variations constatées selon les départements en ce qui concerne le nombre de contrats d'insertion établis et leur contenu en témoignent. Voir par exemple CERC (1992). 
mesure les associations que l'État local finance, qui le renseignent sur les besoins qui se font jour au quotidien sur le terrain. Or, ce n'est pas céder à une vision machiavélique des rapports interinstitutionnels que de remarquer que celles-ci ont plutôt intérêt à diagnostiquer les problèmes en fonction des solutions qu'elles ont à proposer, ce que nombre d'entre elles feront avec d'autant plus de facilité que l'élasticité de la notion d'insertion, nous y reviendrons, rend possible le jeu autour de la qualification des problèmes à résoudre. À côté de cette « connaissance du terrain », une autre ressource est fournie aux associations par la spécialisation dans une activité spécifique et que les institutions publiques se verraient en peine de développer en interne. De nombreuses associations proposent ainsi des services très « pointus » et revendiquent une expertise en la matière. Ainsi, telle association qui propose des « stages de redynamisation sociale et d'approche individualisée de la vie professionnelle », telle autre une « aide aux démarches administratives pour les allocataires du RMI âgés de plus de 55 ans », telle autre un « accompagnement psychosocial d'allocataires du RMI séropositifs ou atteints du sida $»^{1}$. Il ne nous appartient pas de juger de la réalité des compétences revendiquées et encore moins du contenu des activités proposées. Tout au plus fera-t-on observer qu'il existe ici un marché au sein duquel s'ajustent une offre et une demande de financement, une offre et une demande d' « activités d'insertion » et que, de toute évidence, offre et demande se rencontrent dans l'intérêt commun bien compris des divers protagonistes : les associations - parfois les cabinets de consultant - n'ont guère de peine à identifier des « créneaux » pour peu que les institutions publiques aient tendance à les voir comme autant de « trous » dans le filet de protection qu'elles proposent. Enfin, certaines associations parviennent, parce qu'elles multiplient les agréments et les missions de service public, à intégrer le noyau dur du partenariat local en matière d'insertion ou, plus largement, d'action sociale locale. Dans les grandes villes, il n'est pas rare que certaines associations d'action sociale emploient plusieurs dizaines voire plusieurs centaines de salariés, brassent des budgets très conséquents et, de par l'importance des activités qu'elles développent, deviennent membres plus ou moins officiellement du cercle fermé des institutions entre lesquelles se négocient au plus haut niveau les grandes orientations de la politique d'action sociale locale.

Cette montée en puissance d'un monde associatif professionnalisé et détenteur de compétences techniques n'est pas sans soulever débats et controverses au sein du milieu politico-administratif où l'on pointe parfois le risque que les associations se substituent aux services publics ou celui d'une instrumentalisation de l'action publique locale par des acteurs qui auraient parfaitement intégré la logique du « système » et la manière dont ils peuvent en tirer bénéfice. Que l'on s'accorde ou non avec cette position normative, force est de constater

1 Ces exemples sont tirés du document déjà évoqué, L'offre d'insertion, guide pratique à l'usage des instructeurs du RMI. 
que l'ambiguïté qui entoure leur statut de partenaire-prestataire n'est pas sans favoriser les jeux de pouvoir et d'influence autour de la distribution des rôles au sein du « marché » de l'insertion ${ }^{1}$. Ajoutons que dans le cas du RMI, mais c'est vrai aussi dans bien d'autres dispositifs, le législateur a jugé préférable de laisser ouverte la liste des institutions susceptibles d'être associées au partenariat ${ }^{2}$. Localement, en dehors des « partenaires contraints ${ }^{3}$ que sont les conseils généraux, les CAF et les MSA, la liste est donc longue des candidats ou des participants potentiels au RMI. L'éventail des combinaisons possibles et la diversité des formes d'implication envisageables sont ainsi à la mesure de l'imprévisibilité des résultats de jeux d'alliance et de luttes autour de chasses gardées qui ne sont jamais totalement stabilisés.

\section{Des problèmes aux frontières floues}

Ce caractère imprévisible est d'autant plus grand que dans un domaine comme celui de l'insertion sociale et professionnelle, les frontières des problèmes qu'il s'agit de traiter sont particulièrement floues. Dans le cadre de la lutte contre l'exclusion, la politique d'insertion a à voir avec la santé, l'éducation et la formation professionnelle, la famille, l'emploi, l'immigration, la banlieue, etc. La définition des problèmes que la politique d'insertion a en charge de traiter est donc ouverte aux arguments de ceux qui, plaidant pour leur cause, proposeront d'intervenir sur le plan sanitaire car « un individu en mauvaise santé ne peut véritablement s'insérer »; feront valoir leur compétence en matière de formation professionnelle puisque «l'insertion sociale passe par l'insertion professionnelle »; argueront de leur connaissance des populations immigrées et des problèmes spécifiques qu'elles rencontrent car « la question de l'insertion ne se pose pas dans les mêmes termes pour les populations issues d'autres cultures »; etc. Sur le plan de l'organisation du partenariat, le caractère extensible de la définition des objectifs poursuivis en matière d'insertion ouvre donc l'éventail des possibles concernant la qualification des problèmes à résoudre et, partant, concernant l'éventail des solutions appropriées. Les interdépendances sont donc fortes mais elles s'expriment dans un contexte dans lequel l' « hybridation » des problèmes est de mise - ou en tout cas accessible à ceux qui y voient une ressource

\footnotetext{
1 Voir à ce sujet Gachet B., (1998).

2 Loi 88-1088 du $1^{\text {er }}$ décembre 1988, article 34 : « Le représentant de l'État dans le département et le président du conseil général conduisent ensemble et contractuellement l'action d'insertion sociale et professionnelle des bénéficiaires du RMI, avec le concours des autres collectivités territoriales et des autres personnes morales de droit public ou privé, notamment les associations, concourant à l'insertion et à la lutte contre la pauvreté et l'exclusion ». Notons que dans son article 30, la loi 1200 du 18 décembre 2003 reprend pratiquement dans les mêmes termes ce principe d'une ouverture du dispositif au monde associatif : « Le président du conseil général conduit l'action d'insertion sociale et professionnelle des bénéficiaires du RMI. Il bénéficie à cette fin du concours de l'État, des autres collectivités territoriales, des organismes chargés de l'emploi et de la formation professionnelle et des autres personnes morales de droit public ou privé, notamment des associations, œuvrant dans le domaine de l'insertion et de la lutte contre la pauvreté et l'exclusion. »

3 L'expression est empruntée à Robert Lafore (1993).
} 
supplémentaire dans la poursuite d'une stratégie - et l'espace de négociation de leur définition largement ouvert. Le corollaire en est que les légitimités liées à une expertise en matière d'insertion peuvent rarement être fondées sur des critères objectivables. En effet, si la plupart des activités proposées en matière d'insertion peuvent être mesurées sous un certain angle (nombre d'usagers pris en charge, nombre de stages effectués, etc.), il demeure toujours difficile d'en donner une mesure à l'aune des résultats qu'elles produisent en terme d'insertion proprement dite, sauf à mobiliser comme ultime critère le nombre d'usagers qui « sortent du dispositif RMI » au cours d'une période donnée - auquel cas il restera, d'une part, à déterminer à qui l'on doit ces « sorties », d'autre part, à se mettre d'accord sur l'idée qu'en donnant un emploi aux usagers, on a réglé le problème de leur insertion. Certaines associations s'appuient sur cette incertitude concernant l'évaluation des résultats des actions d'insertion sociale, pour mettre en avant leur capacité à produire des résultats mesurables, par exemple en s'engageant à faire baisser le nombre de rmistes dans le département de tel ou tel taux au cours d'une période déterminée. Utilisant des méthodes de management issues du secteur de la vente, recrutant des « commerciaux » qu'elles font travailler sur objectifs, ces associations ne cherchent pas à masquer leurs différences avec les méthodes traditionnelles du travail social, lorsqu'elles ne fustigent pas ouvertement l'obsolescence de ces dernières en matière d'insertion professionnelle. Rencontrant les attentes de financeurs qui souhaitent disposer de « résultats concrets », leurs pratiques s'inspirent souvent des méthodes de la psychologie sociale pour faire se rencontrer des employeurs supposés rétifs face à un public stigmatisé (les rmistes) et des usagers qu'il convient de « mettre en situation de travail » plutôt que d' ' assister » comme les travailleurs sociaux sont accusés de le faire. Loin de réduire cependant l'incertitude concernant la définition des problèmes que le RMI est supposé combler, l'apparition de ces nouvelles méthodes réactive au contraire les débats à ce sujet. En premier lieu, on observe des controverses sur la responsabilité de chacun dans l'accès à l'emploi des rmistes. Est-il le résultat du travail de «réassurance psychologique» assuré par les assistantes sociales ou de l'approche proposée par ce type d'association? En second lieu, tout le monde n'est pas d'accord pour considérer que l'accès à l'emploi constitue le signe objectif de l'insertion sociale réussie. A-t-on réglé les problèmes psychologiques, identitaires, relationnels ou familiaux des « exclus » lorsqu'on leur a offert un emploi ? Ces deux points de débat révèlent en creux deux incertitudes plus fondamentales concernant la définition de l'insertion comme objet d'action publique, soit respectivement : l'incertitude quant aux leviers sur lesquels il faudrait agir pour favoriser l'insertion professionnelle, c'est-à-dire finalement l'incertitude sur les causes de l'«exclusion»; l'incertitude quant à la définition de l'insertion réussie, c'est-à-dire l'incertitude sur les objectifs poursuivis par les politiques d'insertion ${ }^{1}$. Au-delà, ce

1 Sur l'émergence de tels débats entre acteurs du dispositif RMI, voir I. Astier (1997). 
sont les formes d'implication des divers acteurs que le flou de la notion d'insertion rend négociables, chacun étant toujours en mesure de promouvoir une définition qui, allant dans le sens de ses intérêts, lui permettra soit d'argumenter du bien-fondé de sa participation au partenariat, soit de justifier sa non-implication dans le dispositif ${ }^{1}$.

Le RMI n'est bien sûr ici qu'un exemple. Mais il illustre de manière éclairante le lien qui existe entre le caractère transversal ou indivisible des problèmes et la dépendance mutuelle qui unit les acteurs locaux. Dès lors que certains acteurs peuvent utiliser l'élasticité de la définition des problèmes à résoudre comme une ressource disponible pour se porter candidat à l'action collective - démarche dont le succès est lié à la capacité, inégalement distribuée, à imposer la légitimité de la définition que l'on mobilise - il n'est pas surprenant que les acteurs de la lutte contre l'exclusion soient aussi nombreux que sont multiples les manières de définir ce qu'est l'exclusion et ce que doivent être les réponses appropriées en la matière. Face à des problèmes qui reçoivent autant de définitions qu'il est d'acteurs s'estimant concernés, chacun, au sein de configurations d'acteurs évolutives et quels que soient son statut, ses attentes, son autorité ou son pouvoir, doit tenir compte des autres, lesquels sont liés par le comportement de chacun. Le risque - si l'on nous permet l'usage quelque peu normatif de ce terme c'est, en l'absence d'éléments qui permettraient de " durcir » l'évaluation des résultats des actions entreprises, de voir les règles qui régissent les échanges entre partenaires devenir l'objet central des négociations, le ressort principal de pratiques centrées sur la recherche de conditions d'échanges favorables, au détriment de la réflexion commune sur le produit de ces échanges en termes de construction d'action publique ${ }^{2}$. Et ce glissement vers des formes de coopération qui escamotent la question des finalités concrètes de l'action collective au profit de la négociation permanente des règles d'échange entre partenaires - que l'on pourrait traduire en langage psychosocial par le passage à une centration du groupe sur lui-même plutôt que sur la tâche - peut d'autant plus facilement s'opérer qu'il ne revêt pas nécessairement les formes du conflit et ne mène pas systématiquement à des situations de blocage, mais qu'au contraire il peut sous-tendre la construction de partenariats se donnant à voir comme des formes apaisées d'action collective, présentant tous les signes extérieurs de la bonne entente entre partenaires. Il n'est ainsi pas rare que, face à des actions dont le succès

1 L'instrumentalisation de l'ambiguïté pouvant bien évidemment se faire dans les deux sens : une CAF justifiera sa volonté de se « désengager » (dans la limite des contraintes légales qui lui sont imposées) du RMI par sa centration sur la famille ; une association pourra, en vertu de son implication dans l'action en faveur de la famille, se porter candidate à une participation au RMI.

2 Comme le montre Friedberg (op. cit.), cette autonomisation des échanges politiques peut d'autant plus facilement se réaliser que les partenaires sont en mesure d'externaliser les conséquences de leurs choix. Or, dans le domaine de l'action sociale, on sait qu'il n'est guère d'équivalent de ce que dans le domaine des partenariats industriels et commerciaux on nommerait la « sanction du marché », c'est-à-dire l'existence de mécanismes susceptibles de rappeler aux partenaires qu'ils peuvent être eux aussi les victimes des choix collectifs opérés. 
n'est guère mesurable, le marchandage devenant, parfois à l'insu même des acteurs, le moteur essentiel des relations que les uns et les autres doivent entretenir pour rendre de telles actions possibles, l' « excellence formelle du partenariat » devienne en soi l'objectif de la coopération et le principal critère de son évaluation ${ }^{1}$.

\section{- Hétérogénéité du référentiel de coordination territorialisée dans l'action sociale}

Il est un autre facteur d' « autonomisation des échanges politiques » entre partenaires, qui puise à une autre source d'incertitude et en renforce les effets. Celui-ci est lié à la multiplicité des argumentaires avancés sur la scène politico-médiatique et dans les textes qui encadrent la mise en œuvre des politiques d'action sociale pour démontrer ou faire admettre le bienfondé de la démarche partenariale dans ce domaine. Cette diversité des registres de justification proposés aux acteurs locaux, en accentuant l'hétérogénéité du « référentiel » des politiques d'action sociale ${ }^{2}$, accroît l'incertitude sur les « raisons » de l'injonction à la mise en cohérence de l'action des divers acteurs et à la construction, par cette voie, des problèmes à résoudre. Elle crée ainsi un espace supplémentaire de négociation des modalités d'organisation de l'action collective en faisant entrer dans le champ de ce qui se négocie, les « raisons » pour lesquelles il est préférable d'organiser en commun ce qui jusque-là, officiellement du moins, se concevait de manière cloisonnée.

\section{Six argumentaires entremêlés}

L'histoire récente des politiques d'action sociale fait apparaitre six argumentaires principaux, proposés aux acteurs locaux comme éléments de légitimation d'une approche locale et partenariale de la construction des problèmes que l'action sociale doit entreprendre de gérer.

\section{Adaptation de l'action sociale aux réalités locales}

Tout d'abord, la nécessité d'adapter les contenus de l'action sociale aux " réalités locales » se retrouve dans l'ensemble des secteurs de l'action publique à travers les mouvements successifs de déconcentration et de décentralisation, mais aussi dans de nombreux textes législatifs, par exemple en matière d'aménagement du territoire ou, plus largement dans le

\footnotetext{
1 Nous faisons concrètement référence à la tendance à n'évaluer la qualité d'un partenariat qu'à l'aune de la qualité des relations entretenues par ses membres, quasi indépendamment de ce que ce partenariat permet en termes de contenu d'action publique.

2 L'usage du concept de référentiel reprend la formulation établie par Jobert et Muller (1987).
} 
cadre de la réforme de l'État ${ }^{1}$. Cet argument renvoie essentiellement à des questions d'efficacité de l'action publique mais il sert parfois une conception de cette dernière qui affirme la nécessité de donner un « gouvernement local » à des territoires que définissent une histoire, des traditions, voire une culture. En matière d'action sociale, le premier aspect renvoie à la nécessité d'adaptation aux données socio-économiques locales, ce qui passe, d'une part, par la production de connaissances des problèmes spécifiques des territoires dont les acteurs ont la charge, d'autre part, par la capacité à mesurer l'efficacité des actions entreprises. Ces deux démarches, observation et évaluation, tendent à prendre une importance considérable dans le répertoire des arguments qui justifient l'approche partenariale. L'idée est ici que la coopération et la coordination entre acteurs peuvent être mises au service d'une meilleure connaissance des publics et de leurs besoins, et d'une meilleure adéquation entre ces besoins et les réponses apportées ${ }^{2}$. Le second aspect renvoie non plus à l'adaptation mais à la production des politiques sociales au niveau local.

\section{Promotion de l'expérimentation}

La promotion de l'expérimentation, à l'initiative de l'État comme des collectivités territoriales, constitue une des dispositions importantes de la loi constitutionnelle du 28 mars 2003. Le partenariat territorialisé est, dans ce cadre, valorisé du fait des solutions originales qu'il est susceptible de faire émerger. Cette attente explicite d'inventivité, voire d'imagination - s'appuyant sur une « connaissance du terrain »-, se retrouve dans de nombreux secteurs où elle est une des caractéristiques des politiques « constitutives ». Dans l'action sociale, se verront ainsi encouragées les « pratiques innovantes » en matière d'insertion, les « solutions originales » en matière d'hébergement, etc. ; de même que seront diffusées à l'ensemble des administrations sociales locales les pratiques des DDASS les plus « dynamiques » à cet égard. Le partenariat local est ici placé au service de l'innovation ${ }^{3}$.

\footnotetext{
1 Voir par exemple la loi d'orientation relative à l'administration territoriale de la République (loi n ${ }^{\circ}$ 92-125 du 6 février 1992), qui justifie très clairement l'approfondissement de la déconcentration par le souci d'une « gestion de proximité » plus attentive aux spécificités locales, et plus récemment, la loi constitutionnelle 2003-276 du 28 mars 2003 relative à l'organisation décentralisée de la République.

2 Voir par exemple l'usage de la notion de « partenariat d'observation » que fait l'ODAS dans un rapport consacré à la décentralisation de l'action sociale (ODAS, 2003).

3 Reste alors aux acteurs locaux à promouvoir la pertinence des solutions qu'ils proposent dans le cadre de cette incitation à l'innovation. En effet, les administrations centrales se réservent la possibilité de trier les actions proposées, d'en financer certaines et d'autres non. D'où tout le travail de « promotion » auquel se livrent les acteurs locaux pour faire valoir l'intérêt ou mieux, la nécessité de mettre en œuvre les solutions qu'ils proposent. Ce travail passe souvent par un « court-circuitage » des voies hiérarchiques officielles, ce qui n'est pas pour simplifier les relations déjà complexes entre le «local » et le «national » (Camus, Corcuff, Lafaye, 1993).
} 


\section{Réponse à l'urgence}

Une troisième argumentation en faveur du partenariat se fonde sur la gravité d'un « fléau social » qui impose de ne rejeter aucune solution a priori, mais au contraire de favoriser la contribution de tous ceux que réunit cette « cause commune ». Au nom de l'urgence, toutes les bonnes volontés sont acceptées, et il est impératif de se regrouper face au combat qui est imposé à l'ensemble de la société. Ainsi en est-il de la rhétorique liée à la thématique de l'exclusion au plus fort de la crise économique. Cette thématique, en particulier sur la scène politico-médiatique, s'est construite sur ce principe d'un consensus nécessaire et - non sans puiser au registre de l'émotion autour d'une métaphore guerrière (Frétigné, 1999).

\section{Territorialisation des problèmes sociaux}

Le quatrième argumentaire fonde le territoire comme catégorie de perception des problèmes sociaux. L'illustration la plus évidente en est fournie par le « problème des quartiers ", soit l'idée que certains territoires concentrent les problèmes et qu'il faut par conséquent y concentrer toutes les formes d'action sociale et y réunir tous les acteurs de l'action sociale. Mais l'autonomisation du " problème des banlieues » comme problème public appelant une réponse publique appropriée sous-tend également l'idée qu'il est autre chose que la somme des problèmes plus « classiques » (pauvreté, échec scolaire, chômage...) qui s'y développent. Ce qui marque et surdétermine l'image de la banlieue comme objet d'action publique c'est l'absence de cohésion, de lien social, voire de démocratie, c'est l'absence d'intégrateurs qui relieraient l'espace public des cités à l'ensemble de la collectivité, ce à quoi il faut répondre par une réintroduction de la société incarnée par l'ensemble de ses services publics et au-delà par l'ensemble des acteurs censés assurer la socialité contemporaine (entreprises, commerçants, mais aussi forces de l'ordre, etc.). Il en va ainsi des nombreux dispositifs qui se sont succédé dans le cadre des politiques de la ville et qui ont érigé le partenariat étendu comme modèle de prise en compte transversale et territorialisée des problèmes sociaux. Cette transversalité est appliquée également aux questions liées à l'interculturalité qui jusque-là ne trouvaient pas d'expression dans la problématisation des thèmes d'intervention publique ${ }^{1}$.

\section{Simplification et rationalisation}

Un cinquième élément de cette « rhétorique » du partenariat consiste à affirmer comme nécessaires la mise en cohérence et la coordination des diverses interventions en matière d'action sociale afin de réduire la

1 Comme le montre très clairement Christian Rinaudo (1999), la banlieue est bien une « catégorie urbaine ethnicisée ». 
complexité du « système d'aide » existant. Là aussi, deux axes d'argumentation se combinent :

- la rationalisation de l'offre d'action sociale (d'insertion, d'aides financières, etc.) qui s'appuie sur l'idée que les réponses aux problèmes existent, mais qu'elles sont mal réparties, parfois mal utilisées, en tout cas leur articulation mal organisée ${ }^{1}$. La protection sociale de droit commun (la sécurité sociale) est en elle-même déjà suffisamment complexe - Bruno Jobert (1990) parle d'un « ensemble baroque et ingouvernable » - pour qu'on y ajoute celle d'une action sociale qui multiplie et superpose les aides en tous genres. Il faut coordonner ces actions afin de permettre une plus grande " visibilité » de l'action sociale (que peine à obtenir le ministère des Affaires sociales) ; éviter les redondances, c'est-à-dire savoir localement « qui fait quoi », diagnostiquer les manques, les « trous » du filet de protection, les besoins non ou mal couverts en organisant la concertation locale et la mise à plat du système en mettant tout le monde autour d'une table afin de favoriser l'interconnaissance. Une des questions qui se posent cependant, et non des moindres, est de savoir si l'on ne crée pas de la complexité supplémentaire lorsqu' on multiplie justement ces instances de coordination. Cette question - où est le point d'équilibre au-delà duquel la volonté de simplifier crée de la complexité ? - est aujourd'hui au cœur de nombreux débats d'experts ;

- les désagréments qu'occasionne cette complexité à l'usager lui-même. L'usager de l'action sociale est, par définition, une personne déjà fragilisée à qui on demande de comprendre les rouages et la logique d'un système que les spécialistes reconnaissent eux-mêmes comme peu compréhensible, et à qui, du fait du cloisonnement institutionnel et du manque de coordination entre les différents acteurs, on impose de véritables « parcours du combattant » lorsqu'il s'agit d'obtenir une aide. Toutefois cette complexité est souvent dénoncée dans la littérature spécialisée, y compris par l'inspection générale des Affaires sociales elle-même ${ }^{2}$. Cependant, les projets récurrents de « maisons du social », de « plates-formes d'action sociale » ou le fameux « guichet unique »- évoqué par la plupart des ministres des Affaires sociales mais qui, de toute évidence, se heurte à des frottements organisationnels quand ce n'est pas la réticence des acteurs locaux - font écho à cette argumentation.

\section{Participation des usagers}

Enfin, le dernier argumentaire se fonde sur l'idée de citoyenneté. Il promeut l'extension du partenariat aux usagers eux-mêmes. Il prône la participation, la « démocratie sociale », soit l'idée que la citoyenneté ne trouve pas son

1 Il va sans dire que l'idée de mieux «maîtriser» les dépenses correspondantes n'est pas absente de ce souci de rationaliser l'offre d'action sociale.

2 Voir par exemple les remarques d'Hélène Strohl (1988), pour qui les démarches à effectuer sont d'autant plus lourdes que les usagers sont fragilisés et démunis, tout se passant comme si le poids des efforts à accomplir constituait la contrepartie de l'aide que leur apporte la collectivité. 
origine que dans l'association politique mais aussi dans l'appartenance à la société, médiatisée ici par une commune implication dans les décisions qui affectent les mécanismes de redistribution (Madec, Murard, 1995). Le principe de gestion paritaire de la sécurité sociale en est une illustration (Palier, 2001). Dans le domaine de l'action sociale, il se concrétise dans certains programmes visant à permettre l'expression publique des habitants dans les quartiers défavorisés (associations de locataires...) ou dans la reconnaissance de certaines associations d'usagers comme interlocuteurs légitimes des pouvoirs publics ${ }^{1}$. Mais une autre vision de la participation existe, plus ambitieuse, qui consisterait à « produire localement des politiques publiques qui seraient en même temps des politiques de production de territoire et de cohésion sociale, fabriquant de l'intégration et de l'appartenance par la démocratie » (Autès, op. cit., p. 134). Dans cette perspective, le partenariat institutionnel constitue l'instrument d'un projet d'ampleur, celui d'un « gouvernement local », et son autonomisation, dès lors qu'elle intégrera la participation des «usagers-citoyens », une étape vers une "démocratie renouvelée ».

\section{Pourquoi agir ensemble?}

Les différents arguments qui justifient la démarche partenariale dans l'action sociale locale - l'adaptation aux réalités locales, l'expérimentation, la réponse à l'urgence, la territorialisation des problèmes, la réduction de la complexité et l'accès à la citoyenneté - sont bien évidemment mêlés dans le discours politico-médiatique et dans les textes officiels d'où ils émanent et, dans ce sens, ils constituent les éléments d'une typologie qui répond aux besoins de l'analyse. Ces éléments de légitimation - qui constituent autant de sujets d'interrogation chez les hauts fonctionnaires de l'administration centrale, de sujets de débats entre acteurs locaux et de thèmes de réflexion pour les sociologues de la question sociale - concourent, en posant la question des « raisons » de l'action collective locale, à ouvrir encore, à rendre plus incertaine, la question de ses finalités. Ils ajoutent aux questions traditionnelles que pose l'organisation de tout partenariat - que faisons-nous ensemble et comment allons-nous le faire - une incertitude liée à une question tout aussi centrale pour l'action collective : pourquoi est-ce mieux d'agir ensemble que séparément?

Cette question est centrale du point de vue de l'organisation de l'action collective car les diverses réponses qui y sont apportées constituent autant d'éléments de structuration d'une telle action. La pluralité de réponses disponibles, c'est-à-dire mobilisables par les acteurs, crée de la marge de

1 Le principe de la participation renvoie à la figure de l' « usager-citoyen », promue dans de nombreux textes officiels mais qui peine à s'inscrire concrètement dans l'univers politico-administratif (Lafore, 1992). En matière d'action sociale, l'implication des usagers pose tout particulièrement la question des frontières entre action politique et action sociale, participation et militantisme (Madec, Murard, 1995). 
négociation sur les finalités de l'action collective. Elle offre des ressources à certains qui sont autant de contraintes pour d'autres. Elle crée, pourrait-on dire, de l'espace de jeu, et tend par-là à accroître la probabilité d'une autonomisation des règles d'échange entre des partenaires déjà confrontés à la gestion de problèmes dont la définition est élastique. Le processus d'autonomisation emprunte d'ailleurs les mêmes mécanismes - en en renforçant le poids - que ceux que tend à favoriser l'indivisibilité des problèmes : plus le nombre de « raisons d'agir collectivement » mises en circulation, donc potentiellement légitimes, est important, plus grande est la possibilité, pour les acteurs qui disposent des ressources pertinentes, d'en instrumentaliser l'usage en vue d'une (re)négociation des modalités des échanges qu'ils entretiennent avec leurs partenaires. Ainsi, il n'est pas indifférent qu'à une instance comme la CASU, certains départements aient donné l'objectif de simplifier les démarches assurées par l'usager pour obtenir des aides financières exceptionnelles, pendant que d'autres en faisaient l'instrument d'une refonte totale de l'offre locale d'insertion sociale. On a pu montrer qu'à ces deux options correspondaient deux manières différentes de gérer collectivement les opportunités stratégiques offertes par la création d'un nouveau dispositif qui proposait de multiples interprétations des raisons justifiant la coordination des actions menées en matière d'urgence sociale (Frigoli, 2001).

Il ne s'agit pas ici, au nom d'une lecture de l'action sociale locale en termes d'action collective, de priver les acteurs de tout sens de l'intérêt général ${ }^{1}$, mais bien de mettre en lumière l'une des conséquences de leur capacité à investir stratégiquement l'incertitude injectée dans les systèmes d'action locaux par des administrations centrales et des élites politiques qui érigent la négociation des raisons de l'action publique en mode légitime de construction des politiques publiques.

\section{- Conclusion}

Nous avons essayé de montrer que le caractère transversal des problèmes à traiter, associé à l'existence d'une pluralité de raisons d'agir collectivement, pouvait favoriser dans le secteur de l'action sociale ce que Friedberg nomme l'autonomisation des échanges politiques entre partenaires, c'està-dire l'émergence de formes d'action collective dans lesquelles ne tendent plus à agir comme source de régulation que les volontés conjuguées des acteurs de ne pas être perdants dans les échanges qu'ils nouent avec les autres.

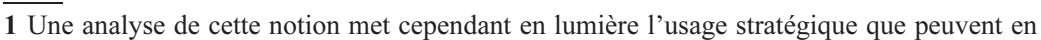
faire les acteurs. (Lascoumes, Le Bourhis, 1998). 
En guise de conclusion, on rappellera qu'en aucun cas il ne s'agit par-là de sous-entendre que les acteurs de l'action sociale seraient intrinsèquement davantage enclins à se comporter en " stratèges » que ceux qui interviennent dans d'autres secteurs. Ici peut jouer un effet d'optique, dont on se gardera d'être dupe, alimenté par le contraste existant dans le domaine du social entre l'intervention de considérations stratégiques et l'omniprésence de discours empreints de compassion à l'égard des personnes en difficulté. Pour le dire autrement, on ne verra dans le constat que nous faisons ni une mise en accusation, ni même un motif de s'émouvoir de ce que des pratiques qui se donnent souvent à voir comme " désintéressées » ou relevant d'un « engagement », puissent partiellement puiser à des ressorts « bassement » stratégiques. Ni plus ni moins « assoiffés de pouvoir » ou « intéressés » que d'autres acteurs, les acteurs du social sont simplement amenés, peut-être plus que dans d'autres domaines, à construire collectivement les problèmes qu'il leur revient de résoudre et les raisons qui justifient de procéder de la sorte. Et il n'y a finalement rien d'étonnant à ce que, dès lors qu'est offerte la possibilité d'un tel travail autour du sens de l'action sociale, les enjeux proprement stratégiques soulevés par la nécessité de s'organiser puissent par moments sembler prendre le pas sur toute autre considération.

Certes, les effets pervers du « copilotage » n'ont pas échappé aux élites politiques et administratives et l'on peut sans doute y voir l'un des ressorts de l'attribution de la gestion de l'ensemble du dispositif RMI aux conseils généraux ${ }^{1}$. Toutefois, tout semble indiquer que, conditionnée à une plus grande "lisibilité des actions » et à une clarification des rôles (ODAS, 2003), la poursuite de la décentralisation sera appelée à voir se maintenir le recours à la démarche partenariale dans le secteur de l'action sociale ${ }^{2}$. Dans cette perspective, il y a lieu d'espérer que le renforcement de l'évaluation et le développement de l'expérimentation, comme pratiques récemment valorisées par les pouvoirs publics, permettront de contenir les risques que nous avons ici tenté de mettre en évidence.

\footnotetext{
1 Voir par exemple le rapport présenté au nom de la commission des Affaires sociales du Sénat dans le cadre des travaux préparatoires à la réforme du RMI (Seillier, 2003). On pourra également se reporter au rapport Mauroy (2000) qui attribue au «système de partenariat » le « brouillage des compétences ».

2 Le principe de «subsidiarité », mis en avant par de nombreux textes (voir par exemple le rapport Delevoye, 1997), n'est pas contradictoire avec l'idée d'un développement de la coordination institutionnalisée entre acteurs locaux. Destiné à faire en sorte qu'existe un " chef de file » pour chaque domaine ou territoire d'intervention, il ne remet en cause ni l'ouverture au monde associatif, ni la volonté affirmée par les pouvoirs publics de favoriser la coopération entre acteurs au nom d'une meilleure efficacité dans la poursuite d'objectifs « transversaux ».
} 


\section{Bibliographie}

ALDRICH H., (1976), « Resource Dependence and Interorganizational relations », Administration and Society, 7 (4).

ALIDIERES S., (2001), « Du Fonds d'urgence sociale aux commissions de l'action sociale d'urgence : rupture et continuité ", Revue française des Affaires sociales, $\mathrm{n}^{\circ} 1$, janvier-mars.

ASTIER I., (1999), Revenu minimum et souci d'insertion, Paris, Desclée de Brouwer.

AUTÈS M., (1999), Les paradoxes du travail social, Dunod.

BÉHAR D., (2001), « Partenariat et territoire : une nouvelle donne », Informations sociales, $\mathrm{n}^{\circ} 95$.

BORGETTO M., (2003), « Identification, problèmes et enjeux du ciblage : un état des lieux de la question », Informations sociales, $\mathrm{n}^{\circ}$ 108, p. 6-16.

CAMUS A., CORCUFF P., LAFAYE C., (1993), « Entre le local et le national : des cas d'innovation dans les services publics ", Revue française des Affaires sociales, $\mathrm{n}^{\circ} 3$, p. 17-47.

CERC, (1992), Atouts et difficultés des allocataires du RMI, La Documentation française.

CHAMPAGNE P., (1993), « La vision médiatique », in Bourdieu P. (ed.), La misère du monde, Paris, Éditions du Seuil, « Libre examen », p. 61-79.

COMMISSARIAT GÉNÉRAL DU PLAN, (1997), Cohésion sociale et territoires, rapport du groupe de réflexion prospective présidé par J.-P. Delevoye, Paris, La Documentation française.

DAMMAME D., JOBERT B., (1995), « La politique de la ville ou l'injonction contradictoire en politique », Revue française de science politique.

DURAN P., THOENIG J.-C., (1996), « L'État et la gestion publique territoriale », Revue française de science politique, $\mathrm{n}^{\circ} 4$.

FRÉTIGNÉ C., (1999), Sociologie de l'exclusion, Paris, L'Harmattan.

FRIEDBERG E., (1993), Le pouvoir et la règle.

FRIGOLI G., (2001), « Éléments de synthèse sur la mise en place des commissions d'action sociale d'urgence », Revue française des Affaires sociales, $\mathrm{n}^{\circ}$ 1, janvier-mars, p. 185-197.

GACHET B., (1998), « Associations et communes. Analyse des comportements dans une optique public choice », in MiRe, «Rencontres et Recherches », Produire les solidarités, la part des associations, MiRe-Fondation de France, p. 125-141.

GAUDIN J.-P., (1996), « La négociation des politiques contractuelles », in Gaudin J.-P. (dir.), La négociation des politiques contractuelles, L'Harmattan, p. 7-29.

GUYOT J.-C., LAUDOYER P., (1998), « La santé des exclus au risque d'une politique de la ville ", Revue française des Affaires sociales, $\mathrm{n}^{\circ} 3$, juillet-septembre.

IGAS, (2004), Synthèse des bilans de la loi d'orientation du 29 juillet 1998 relative à la lutte contre les exclusions, mai.

ION J., (1990), Le travail social à l'épreuve du territoire, Éditions Privat, Toulouse.

JOBERT B., MULLER P., (1987), L'État en action. Politiques publiques et corporatismes, Paris, PUF.

JOBERT B., (1990), « Mode de médiation sociale et politiques publiques : le cas des politiques sociales ", L'Année sociologique, $\mathrm{n}^{\circ} 40$. 
LAFORE R., (1992), « Droit d'usage, droit des usagers : une problématique à dépasser », in Chauvière M., Godbout T. (dir.), Les usagers entre marché et citoyenneté, L'Harmattan.

LAFORE R., (1993), «Insertion et collectivités locales, le territoire de l'insertion », in Laborde J.-P., Daugareilh I. (dir.), Insertions et solitudes, MSHA.

LASCOUMES P., LE BOURHIS J.-P., (1998), « Le bien commun comme construit territorial », Politix, $\mathrm{n}^{\mathrm{o}} 42$.

LASCOUMES P., (1996), « Rendre gouvernable. De la traduction au transcodage.

L'analyse des processus de changement dans les réseaux d'action publique », in La gouvernabilité, Paris, CURAPP, PUF, p. 325-329.

LÖCHEN V., (2000), Guide des politiques sociales et de l'action sociale, Paris, Dunod.

MADEC A., MURARD N., (1995), Citoyenneté et politiques sociales, « Dominos », Flammarion.

MARCOU G., (1996), « Gouverner les villes par le droit ? », in CURAPP, La gouvernabilité, PUF.

MAUROY P., (2000), Refonder l'action publique locale, rapport au Premier ministre, Paris, La Documentation française.

ODAS, (2003), La décentralisation de l'action sociale, bilan et perspectives, rapport de l'Observatoire national de l'action sociale décentralisée sur l'acte II de la décentralisation.

PADIEU C., SANCHEZ J.-L., (1994), L'action sociale, dix ans de décentralisation, 1984-1994, Paris, ODAS Éditeur.

PALIER B., (2001), « De la démocratie à la refondation sociale », Informations sociales, $\mathrm{n}^{\circ} 95$.

RINAUDO C., (1999), L'ethnicité dans la cité. Jeux et enjeux de la catégorisation ethnique, L'Harmattan.

SEILLIER B., (2003), Décentralisation du revenu minimum d'insertion (RMI) et création d'un revenu minimum d'activité (RMA), rapport 304, tome 1 , Commission des affaires sociales du Sénat.

STROHL H., (1988), « Les exclus et les organismes sociaux », Revue française d'administration publique, $\mathrm{n}^{\circ} 45$, janvier-mars.

THÉVENET A., (1997), L'aide sociale aujourd'hui après la décentralisation, Paris, ESF Éditeurs.

WACQUANT L., (1992), « Pour en finir avec le mythe des "cités ghettos". Les différences entre la France et les États-Unis », Annales de la recherche urbaine, no ${ }^{\circ}$, p. 21-30. 